\title{
On the optimal inner tube shape for an ultrasonic nebulizer
}

\author{
T. E. Stokes G. C. Hocking* \\ (Received 13 July 2000, revised 7 September 2001)
}

\begin{abstract}
We investigate the effect of inner tube shape on the flux of medicine in a Sheiman ultrasonic nebulizer. Using a simple model for the flow of the aerosol up through the inner tube, we consider the variation in flux for cylinders of varying cross-section. The optimal shape is found to be proportional to the shape of the inner jet for any assumed pressure difference between the upper and lower chambers of the nebulizer.
\end{abstract}

*Mathematics and Statistics, DSE, Murdoch University, Murdoch, WA 6152, Australia. mailto:hocking@prodigal.murdoch.edu.au

${ }^{0}$ See http: //anziamj . austms.org.au/V43/E020 for this article and ancillary services, (C) Austral. Mathematical Soc. 2001. Published 9 October 2001. 


\section{Contents}

1 Introduction

E39

2 Liquid Jet Behaviour

E41

3 A model of the flow

E43

4 Constant cross-sectional radii

E48

5 Varying cross-sectional radii

E52

6 Some families of tube shapes

E54

7 The tube optimality condition

E60

8 Optimizing the outer tube for various pressure differences $\quad$ E69

9 Conclusions

E73

References

E74 


\section{Introduction}

A nebulizer is a device for the reduction of some liquid or particulate drug into a form to be inhaled. The process creates droplets of the medicine which are small enough to travel all of the way into the lung without "crashing" into the walls of either the inhalation tube or the throat. There are two common methods for doing this. The first involves the propulsion of a jet of air past a small tube containing the medicine. These so-called air-jet nebulizers draw the medicine from the small tube where it is carried along by the air into a chamber from which the liquid droplets can be inhaled. These devices involve a motor, tubes and an inhalation mask and are rather cumbersome.

The second kind of nebulizer uses ultrasonic radiation passing through a liquid medicine to cause a jet of liquid that breaks up into droplets which can then be inhaled in some way. The 1998 Mathematics-in-Industry Study Group [2] examined a nebulizer which is of the latter type. This device has a different design to earlier models and consists of upper and lower chambers separated by an inner tube (see Figure 1). Liquid medicine in the lower chamber is excited by the ultrasound causing a thin jet, with radius of about 2-3 $\mathrm{mm}$ and an upward velocity around $1 \mathrm{~m} / \mathrm{s}$, to travel up through the inner tube into the upper chamber. This jet begins to break up in the central tube due to cavitation caused by the ultrasonic excitation and the liquid droplets formed here are carried along into the upper chamber. The resulting droplet cloud is forced out through another tube into the mouth of the patient. The entire process is driven by the pressure differences that arise between the 


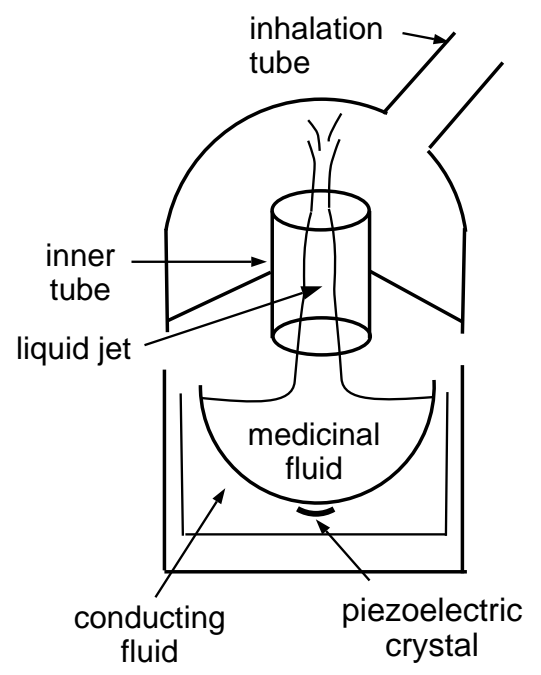

Figure 1: Sketch of the Sheiman Ultrasonic Nebulizer

upper and lower chambers and the outside. Droplets of medicine which do not pass into the inhalation tube drain back from the upper chamber to the lower and thus are not wasted.

There are a number of aspects to the design of these particular devices that have advantages over the older models, but there are still a number of problems that need to be addressed. The major advantage is their compact size and minimal power requirements. A standard small battery can be used to power it and it is small enough to be carried in a hand bag. The problems 
relate to the size of the droplets and the absorbance of particulate medicine into the liquid. A more complete understanding of the processes is required to address these problems.

The MISG was involved in discussing a number of issues. But the issue of interest to this paper is the design of the central tube to optimise the flow of air, thereby maximising drug delivery rate. A simple model is used to consider a range of different shapes for the central tube in an attempt to find an optimal shape.

\section{Liquid Jet Behaviour}

The generation of the liquid jet by the ultrasonic radiation is a particularly interesting problem, but one that we will not consider here. However, the jet of liquid medicine which sprays up through the middle of the central tube is important since it is this which "drags" the air upward and creates the pumping action for the inhalation of the drug. The jet is very narrow and has quite high velocity. It is important to consider the shape of this jet.

Assuming uni-directional flow of a thin circular jet, and hence a velocity independent of radial variable $r$, and ignoring viscosity, the jet must conserve flux:

$$
Q=\pi a(z)^{2} v_{z}=\pi a_{0}^{2} v_{0}, \quad z>0
$$

where $Q$ is the constant upward flux, $v_{z}(a)$ is the upward velocity $\left(v_{z}=v_{0}\right.$ 
at $z=0)$ and $a(z)$ is the radius of the jet $\left(a=a_{0}\right.$ at $\left.z=0\right)$. The pressure at the surface must match atmospheric pressure, leading to a condition given by the Bernoulli equation:

$$
\frac{1}{2} v_{z}^{2}+g z=\frac{1}{2} v_{0}^{2}
$$

Combining (1) and (2) gives an expression for the radius of the jet at any height as

$$
a(z)=\left(\frac{v_{0} a_{0}^{2}}{\left(v_{0}^{2}-2 g z\right)^{1 / 2}}\right)^{1 / 2} .
$$

By examining the denominator of $a(z)$ in $(3)$, see that this solution is undefined if $z=v_{0}^{2} / 2 g$, so a jet with initial velocity of $v_{0}=1.2 \mathrm{~m} / \mathrm{s}$ will continue to a height of about $7 \mathrm{~cm}$. Since for many of the calculations later in the paper we have chosen the height of the inner tube to be $6 \mathrm{~cm}$, this is not a problem.

In reality the situation is even more complicated because of cavitation in the jet itself, and also the presence of air bubbles. To model all of these factors would be extremely complicated, and our interest here is only to get a rough idea of the shape of the central jet as an input for modelling the air flow. Once the jet breaks up, the individual liquid droplets continue to travel upward, but again this is rather complicated to model, and so we make the above assumption (3) about its shape. 


\section{A model of the flow}

Our concern in this paper is the flow of air up through the central tube: is there a size and shape for the tube that maximises the flow rate? The flow begins almost immediately that the ultrasonic crystal is initiated and the liquid jet shoots upward. The cloud of liquid droplets is seen to emerge from the upper chamber equally quickly. Air is drawn in from the outside through vents in the lower chamber and there is a consequent flow out through the mouthpiece from the top chamber.

A model of the outer tube with a narrow, circular cylinder moving rapidly upward through the middle [2] showed that once the jet is established, viscous forces would draw the air upward within the required time frame. A steady flow is established within seconds. Bearing this in mind, we will use a model of almost uni-directional flow [1]. A rigid stationary outer tube of slowly varying radius $R(z)$ and length, $L$, surrounding a smaller "tube" of slowly varying radius $a(z)$ travelling upward with average velocity $v_{z}(r, 0) \approx 1 \mathrm{~m} / \mathrm{s}$ will be used to model the process (see Figure 2). (For comparison, a jet satisfying (3) and having initial velocity $v_{0}=1.2 \mathrm{~m} / \mathrm{s}$ has an average velocity up the length of the tube of $0.9027 \mathrm{~m} / \mathrm{s}$.) Considering the high velocity of the central jet it is a reasonable assumption that the flow will be almost uni-directional [1] provided $R(z)$ and $a(z)$ are changing slowly. The liquid droplets will be assumed to be carried along by the flow of air, and so will not be explicitly incorporated. 


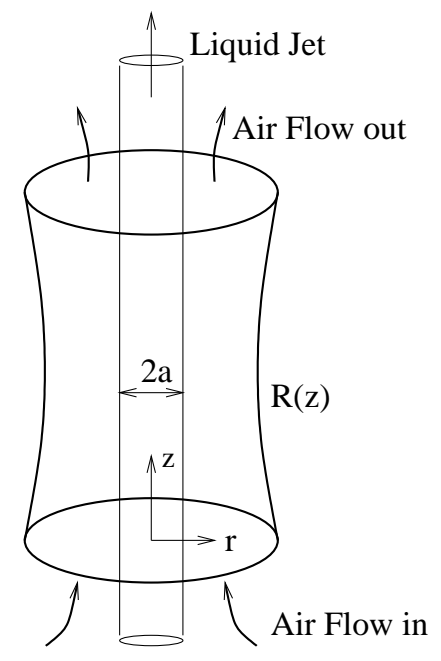

Figure 2: Model for inner tube design 
A factor of major importance is the pressure in the upper and lower chambers and outside of the device, and the flow of air in through the vents and out through the mouthpiece. If the air in the lower chamber is at a higher pressure than that in the upper, i.e. is aiding the flow of air, then the best solution will simply be the widest tube that can be fitted, since the air flux will simply increase with radius. However, since air is drawn in from the outside at the bottom and is expelled from the top chamber into the atmosphere, it is clear that the pressure in the bottom chamber is lower than atmospheric, and the pressure in the upper chamber is higher than atmospheric, and thus is counter to the desired direction of flow of air upwards. One would expect that the pressure in the upper chamber would be lower than atmospheric (since it is moving more quickly) but this is clearly modified by the presence of the medicinal aerosol mist which also occupies the upper chamber, modifying the density.

Clearly the flow generated by the upward jet is strong enough to overcome this adverse pressure gradient, but it does suggest that there may be some optimal radius for the inner tube. It is evident that once the flow develops, the pressure in the two chambers will be modified. A full model of this feedback would be quite difficult especially due to the presence of the mist and we would lose the simplicity of the current model. So for the moment we will assume that the pressure gradient is known and calculate the consequent flow.

Using cylindrical polar coordinates so that the $z$-axis is aligned with the jet, with $z=0$ corresponding to the centre of the bottom of the tube (see 
Figure 2), and assuming uni-directional flow, the Navier-Stokes equations reduce to

$$
\frac{d p}{d z}=\mu \frac{1}{r} \frac{d}{d r}\left(r \frac{d v_{z}}{d r}\right),
$$

where $v_{z}(r, z)$ is the upward velocity of air and $\mu$ is the dynamic viscosity of air. The $r$-momentum equation gives simply that the pressure depends on $z$ only, i.e. $p(z)$, and hence we finish with a differential equation involving the velocity, $v_{z}(r, z)$ and $p(z)$. The continuity equation then provides an extra equation, and given the dependence of the variables this can be integrated to ensure conservation of mass of air moving upward. At any section of the tube it must be true that

$$
Q=2 \pi \int_{a(z)}^{R(z)} v_{z} r d r
$$

where $Q$ is a constant equal to the flux of air past a particular height. On the inner boundary of the outer tube, the velocity of air must be zero, and on the outer boundary of the inner jet, the air is carried along by the liquid. Thus the boundary conditions on the flow are

$$
\begin{cases}v_{z}=0, & r=R(z), \\ v_{z}(r, z)=v(z), & r=a(z),\end{cases}
$$

where $v(z)$ is the upward velocity of the central jet. At this point we have made no assumptions about the form of $R(z), a(z)$ nor $v(z)$.

The problem is to solve equations (4) and (5) with boundary conditions (6). The drawback of this system is that it is not complete. We still do 
not know the pressure distribution and hence cannot compute $d p / d z$. Since $d p / d z$ is constant at a particular value of $z$, we rearrange (4) and solve for $v_{z}$ as a function of $r$ and $d p / d z$ at any $z$. This gives

$$
v_{z}(r, z)=v \frac{\log (r / R)}{\log (a / R)}-\frac{1}{4 \mu} \frac{d p}{d z}\left[\left(R^{2}-r^{2}\right)-\left(R^{2}-a^{2}\right) \frac{\log (r / R)}{\log (a / R)}\right] .
$$

Substituting this into equation (5) gives an expression for $Q$, the (constant) flux as a function of $d p / d z$ and tube radius $R$, namely

$$
Q=h(R)+\frac{d p}{d z} k(R)
$$

where $h(R)=\frac{\pi v}{2}\left[\frac{\left(R^{2}-a^{2}\right)}{\log (R / a)}-2 a^{2}\right]$

$$
\text { and } k(R)=-\frac{\pi}{8 \mu}\left[\left(R^{4}-a^{4}\right)-\frac{\left(R^{2}-a^{2}\right)^{2}}{\log (R / a)}\right] .
$$

Clearly, the flux $Q$ will depend on the pressure difference between the two ends of the tube. We will take the pressure at the top (in the upper chamber) to be $p(L)$ and that at the bottom (in the lower chamber) to be $p(0)$, where $p(0)<p(L)$. This assumption will allow us to examine the flow in the tube for different values of $\delta p=p(L)-p(0)$.

We begin by considering the flow in a tube of constant radius under the assumption that the liquid jet is a cylinder of constant radius in order to 
determine if there is an optimal tube radius. We then consider various other cases and give the jet itself a more realistic shape. We obtain an optimality condition empirically and then verify its correctness, and use it to find the optimal tube shape given any assumed shape of the jet. Since we are unable to compute the actual pressure in the top and bottom chambers using the current model, we will compute the flow over a range of realistic flows and compute the optimal design parameters as a function of the pressure drop between the chambers.

\section{Constant cross-sectional radii}

If we assume that the tube and jet radii do not depend on $z$, that is the equations of the tube wall and the jet boundary are $r=R$ and $r=a$ respectively ( $R$ and $a$ constants), then the flow itself is independent of $z$, and hence $d p / d z$ is a constant which we know is positive, that is, in this case

$$
\frac{d p}{d z}=\frac{\delta p}{L}
$$

where $\delta p=p(L)-p(0)$. Similarly the jet velocity $v(z)$ is a constant, $v_{0}$. Figure 3 shows two velocity profiles $v_{z}(r)$ for fixed values of the parameters, but two different values of $d p / d z$. Importantly, for one case, the flow is upward across the entire width of the tube, but in the other, having a stronger adverse pressure gradient, a small back flow develops in the tube. Thus the 


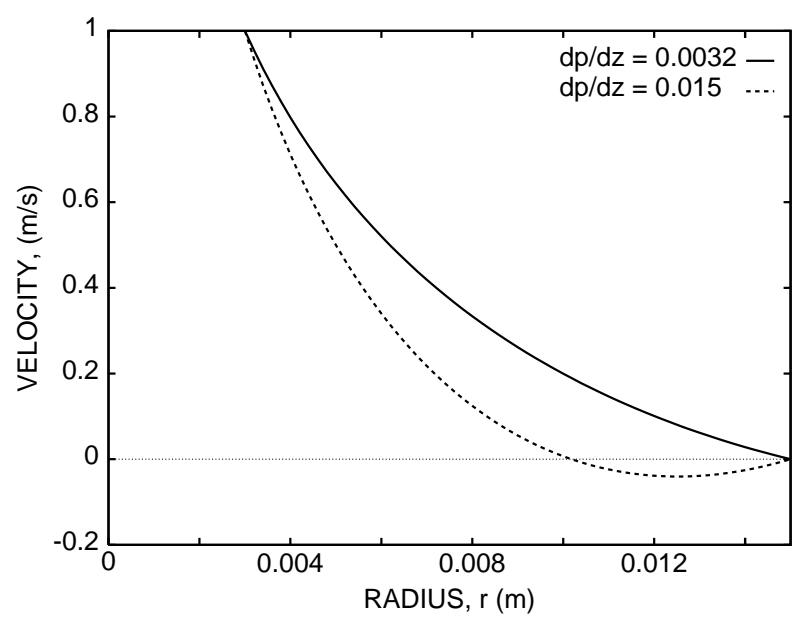

FiguRE 3: Velocity profiles in the tube for two different pressure gradients

air near the outer edge of the tube is actually flowing downward into the lower chamber.

We are interested to see if there is a particular radius $R$ which will give a maximum flux $Q$ for a given value of $d p / d z$. The value of $d p / d z$ is not known exactly, but we can treat it simply as a parameter of the problem. Figure 4 is a plot of $Q$ versus $R$ for the following realistic values of the parameters:

$$
\begin{aligned}
& a=.003 \mathrm{~m}, \quad v_{0}=1 \mathrm{~m} / \mathrm{s}, \quad \delta p=.0032 \mathrm{~Pa} / \mathrm{m}, \\
& \mu=1.8 \times 10^{-5} \mathrm{~kg} / \mathrm{m}, \quad L=.06 \mathrm{~m} .
\end{aligned}
$$




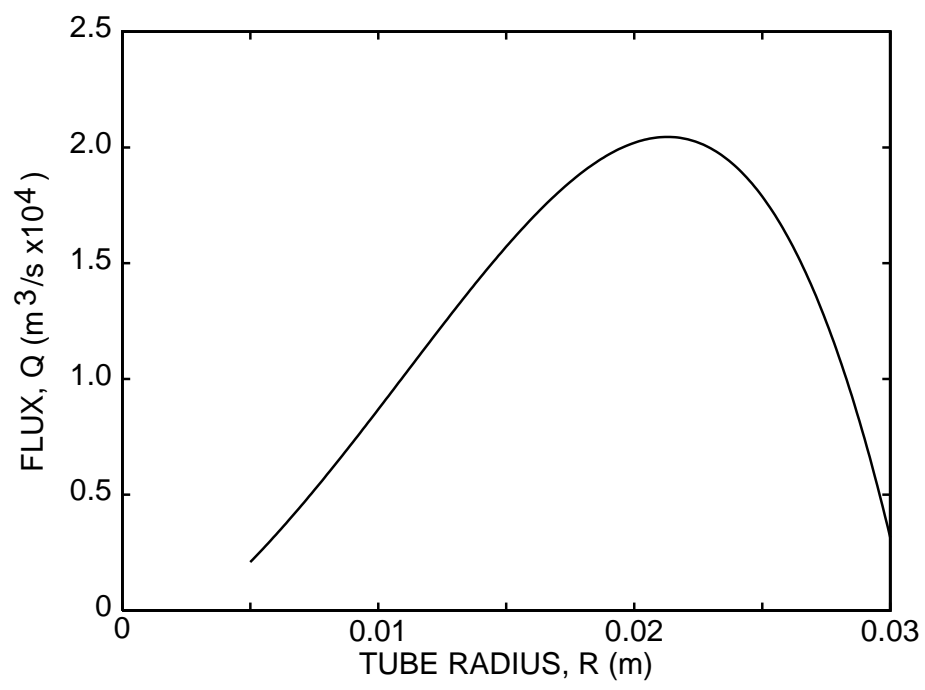

Figure 4: Plot of flux $Q$ versus tube radius $R$

Evidently $Q$ reaches a maximal value when $R=0.021 \mathrm{~m}$ approximately. In general we can find such values by differentiating (8) with respect to $R$ and solving the result put equal to zero. This was done symbolically in Maple, with all parameters left unevaluated, yielding

$$
R_{\max }=a \cdot \exp \left[\frac{1}{2}+\frac{1}{2} \operatorname{LambertW}\left(\frac{4 \mu v-\frac{d p}{d z} a^{2}}{e \frac{d p}{d z} a^{2}}\right)\right],
$$

where LambertW is the Lambert W-function (see [3]). Essentially LambertW 


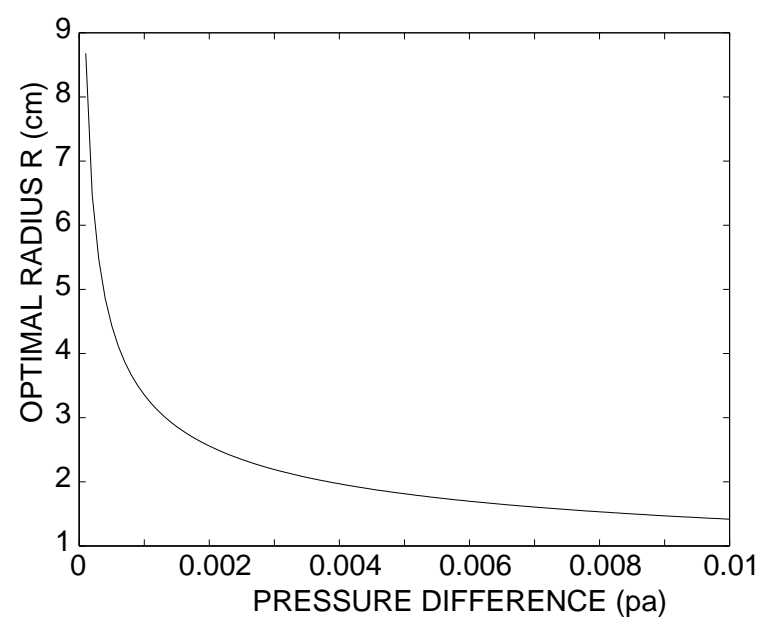

Figure 5: Plot of optimal radius $R_{\max }$ against pressure difference $\delta p$

is the unique, everywhere analytic, branch of the inverse function of $f(x)=$ $x e^{x}+x$. It is defined when its argument exceeds $-e^{-1}$, which here requires $\mu v>0$, which is certainly satisfied.

Of greatest interest is the variation of $R_{\max }$ with respect to $d p / d z$. With the parameter values other than $\delta p$ as in (10), we obtain Figure 5.

See that $R_{\max }$ increases without limit as $\delta p \rightarrow 0$. On the other hand, it drops away asymptotically to a small positive value as $\delta p \rightarrow \infty$, and from (11), the value approached is 0.003 , the assumed radius of the inner jet. 
All of this is to be expected. As the adverse pressure gradient reduces, there is a reduced likelihood of a back flow, and so the optimal radius increases without limit since a wider tube will accommodate more flow. As $d p / d z$ increases, however, there is a greater backflow, and so a smaller radius is necessary to reduce this negative influence on the flux, with the outer tube approaching the inner jet as the pressure gradient becomes more adverse.

\section{Varying cross-sectional radii}

Given that there is an optimal constant radius for the tube if the jet is assumed to be a cylinder of constant radius, a natural question is whether there is an optimal shape, possibly involving a variable radius. Moreover, the jet is not a simple cylinder as we have seen and actually spreads out somewhat from its initial width. To begin to answer this we need to compare different tube shapes having the same "average" radius, for a given jet shape so that size and shape issues can be treated independently and an overall optimum tube shape found. We do this by considering a number of different parametrized families of tubes, in which one parameter is average radius and the other is associated with the shape.

Rearranging (8) we obtain $d p / d z$ on its own:

$$
\frac{d p}{d z}=f+Q g
$$


where $f=4 \mu v\left(\frac{R^{2}-a^{2}}{\log (R / a)}-2 a^{2}\right)\left[\left(R^{4}-a^{4}\right)-\frac{\left(R^{2}-a^{2}\right)^{2}}{\log (R / a)}\right]^{-1}$, and $g=-\frac{8 \mu}{\pi}\left[\left(R^{4}-a^{4}\right)-\frac{\left(R^{2}-a^{2}\right)^{2}}{\log (R / a)}\right]^{-1}$.

Both $f$ and $g$ are functions of $z$, since $R, a$ and $v$ are. Note that $v$ is itself a function of $a$ : the jet velocity reduces as the jet widens in accordance with the conservation of flux condition, so that $v(z)=v_{0}\left[a_{0} / a(z)\right]^{2}$ from (1), where we assume $v_{0}=v(0)=1.2 \mathrm{~m} / \mathrm{s}$ and $a_{0}=a(0)=.003 \mathrm{~m}$.

Now, $Q$ must be constant and therefore the pressure difference between the ends of the tube, $\delta p=p(L)-p(0)$, is given and hence fixed. Then integrating both sides of (12) with respect to $z$ from 0 to $L$ and rearranging gives

$$
Q=(\delta p-F) / G
$$

where

$$
F=\int_{0}^{L} f(R(z), a(z), v(z)) d z, \quad G=\int_{0}^{L} g(R(z), a(z), v(z)) d z .
$$

In this way, given $a(z)$ and hence $v(z)$ via (1), as well as $\delta p$, we view $Q$ as a function of the cross-sectional shape given by $R=R(z)$. Once $R(z)$ is chosen, $Q$ is obtained by numerical integration using Matlab. 


\section{Some families of tube shapes}

Our approach at this point is to consider several families of parametrized functions $R_{m}(z)$ ( $m$ real). For each family, the $m=0$ case represents straight sides of constant radius $R_{0}$, but for all values of $m$, the rule $\int_{0}^{L} R_{m}(z) d z=$ $R_{0} L$ is satisfied; we call such a parametrized family regular. Thus the total area of a cross-section of the tube taken through the middle along the $z$ direction is constant as the shape parameter $m$ changes. The size of the tube is governed by the value of $R_{0}$, whereas its shape is determined by $m$, so that we should think of each family as being parametrized by both $m$ and $R_{0}$. Of course only a certain range of shapes can be represented by a single parameter: hence the need to consider several families. We return to the question of the jet shape $a(z)$ shortly.

The classes of tube shape to be considered are as follows.

1. Straight sides, tapering from bottom to top $(m>0)$ or top to bottom $(m<0)$, see Figure 6. Here we assume $R(z)$ is a linear function of $z$ :

$$
R(z)=R_{0}-m(z-L / 2) .
$$

It is easy to see that this family is regular. Note that $m$ is the slope of the line $R=R(z)$, which passes through the point $\left(L / 2, R_{0}\right)$.

2. Parabolic convex sides, tapering from bottom to top $(m>0)$, and parabolic concave sides, tapering from top to bottom $(m<0)$. Here we 


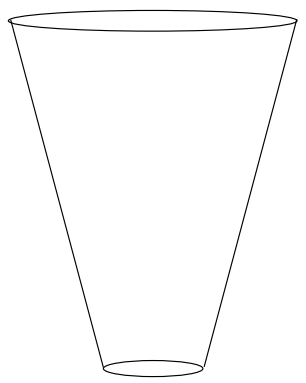

Case 1

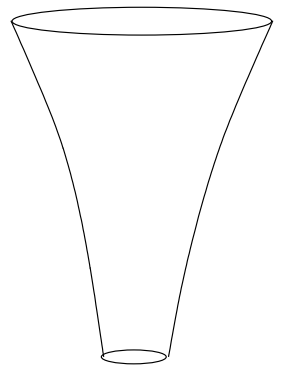

Case 2

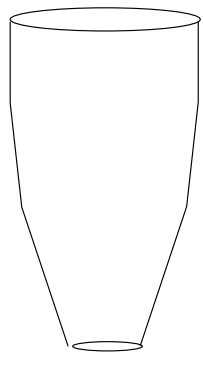

Case 3

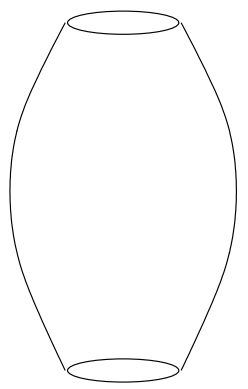

Case 4

Figure 6: Sketch of tube trial shapes

assume $R_{m}(z)=R_{0}-m\left(3 z^{2}-L^{2}\right) / L^{2}$. This form of $R_{m}$ was forced by the assumption that the family be regular. When $m=0$ we obtain $R=$ $R_{0}$ and for larger values of $m$, the function becomes progressively more convex; similarly for negative values of $m$.

3. Parabolic convex sides, tapering from top to bottom $(m>0)$, and parabolic concave sides, tapering from bottom to top $(m<0)$. This time $R_{m}(z)=R_{0}-m\left(3(L-z)^{2}-L^{2}\right) / L^{2}$.

4. Parabolic sides, symmetric, convex $(m>0)$ and concave $(m<0)$. Here we have $R_{m}(z)=R_{0}-m\left(12(z-L / 2)^{2}-L^{2}\right) / L^{2}$.

We have not yet considered the shape of the inner jet. It turns out that a Case 2 tube of the form $a_{m}(z)=a_{0}-m\left(3 z^{2} / L^{2}\right)$, with $m=-.0005$, gives a 


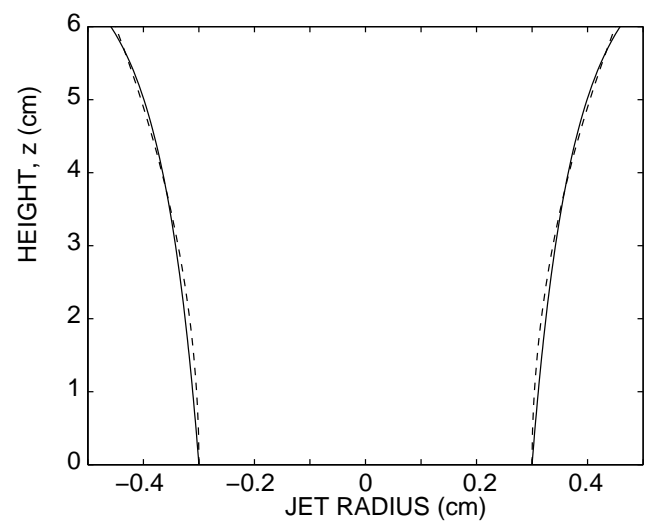

Figure 7: Comparison of the actual jet shape and our approximation

very good fit to the actual jet function $a(z)$ as in Section 2, better even than the three-term Taylor series expansion of $a(z)$ about $z=0$ : see Figure 7 . We use this rather than the actual jet shape as in (3) since it is compared more directly with the optimal outer tube shape we obtain, especially if this turns out to be of Case 2 type as we might expect.

Because the Case $2 R_{m}(z)$ is a regular family, it follows that the $a_{m}(z)$ family is also, having average radius $a_{0}-m$; a value of $m=-.0005$ thus gives an average jet radius of $.0035 \mathrm{~m}$. For the purpose of calculations, we use the conservation of flux condition (1) to obtain $v(z)$, so effectively the Bernoulli 
equation (2) is violated (slightly). We use the values of the various parameters as in (10), except that $v_{0}=1.2 \mathrm{~m} / \mathrm{s}$ now, giving an average velocity for the jet shape as in $(3)$ of around $.9 \mathrm{~m} / \mathrm{s}$.

For this approximation to $a(z)$, we obtained 3-D plots of $Q$ versus $m$ and $R_{0}$ for each of the four cases just discussed: see Figure 8 for Case 2 . In the process we also found the optimal values of $R_{0}$ and $m$ for each. The results are as follows:

Case 1: $R_{0}=2.22 \mathrm{~cm}, m=-.135, Q=2.07 \times 10^{-4} \mathrm{~m}^{3} / \mathrm{s}$;

Case 2: $R_{0}=2.24 \mathrm{~cm}, m=-.0032, Q=2.08 \times 10^{-4} \mathrm{~m}^{3} / \mathrm{s} ;$

Case 3: $R_{0}=2.21 \mathrm{~cm}, m=.0022, Q=2.05 \times 10^{-4} \mathrm{~m}^{3} / \mathrm{s}$;

Case 4: $R_{0}=2.15 \mathrm{~cm}, m=-.0005, Q=1.98 \times 10^{-4} \mathrm{~m}^{3} / \mathrm{s}$.

These results were largely predictable: Case 2 is the closest to the jet shape, at least for $m<0$, followed by Case 1 with $m<0$, and Case 3 with $m>0$, whereas no Case 4 tube is similar to the assumed jet shape.

Of more interest is that, of all cases tested, the optimal $R(z)$ is such that $R(z) / a(z)$ is essentially constant: the optimal tube has

$$
R(z)=.0224+.0032\left[\left(\frac{z}{L}\right)^{2}-1\right]=.0192+.0032\left(\frac{z}{L}\right)^{2},
$$




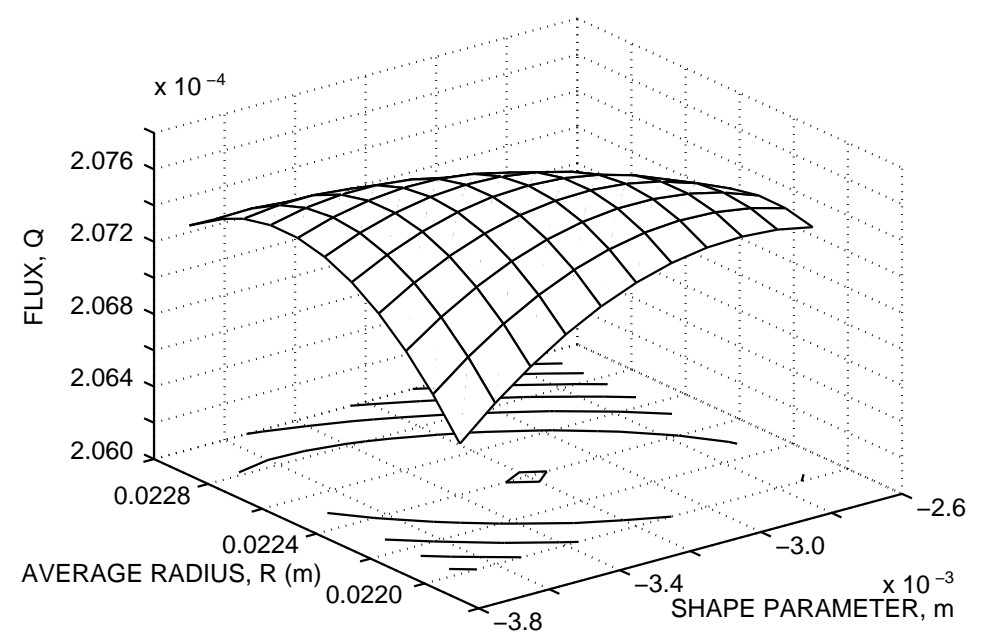

Figure 8: Flux versus parameters $m$ and $R_{0}$ for Case 2 
whereas $a(z)=.003+.0005(z / L)^{2}$, so $R(z) / a(z)=7.47$. By considering other values for the pressure difference between the ends of the tube, $\delta p$, namely $1.6 \times 10^{-3} \mathrm{~Pa}$ and $\delta p=6.4 \times 10^{-3} \mathrm{~Pa}$ and repeating the above process, the optimal $R(z)$ again turned out to be a simple multiple of $a(z)$, although the constant of proportionality varied with $\delta p$. We even considered other less realistic jet shapes and sizes based on our four cases, with $v(z)$ still determined by the conservation of flux condition (1) but not the Bernoulli equation (2), and again the optimal $R(z)$ came out to be a multiple of $a(z)$, although again the constant of proportionality varied with the choice of $a(z)$ even with $\delta p$ fixed. For instance, if the jet was taken to be a cylinder of constant cross-sectional radius as in Section 3, the optimal tube shape amongst all four cases was a cylinder of constant radius equal to that given earlier. On the other hand, using $a(z)$ as in (3), the apparent optimal $R(z)$ was shown empirically not to come from any of Cases 1 to 4 but rather from one of the multiples of $a(z)$ itself.

This leads to an obvious conjecture: that in the optimal situation, $R(z)$ is always a simple multiple of $a(z)$, if the conservation of flux condition is assumed but Bernoulli's equation is not, regardless of the assumed form of $a(z)$ or the choice of parameters such as $\delta p, v_{0}$ and so forth. Note that this conjectured condition does not correspond to equal areas of cross-section at different heights.

We mention incidentally that the condition fails if we require instead that the jet satisfy Bernoulli's equation but not the conservation of flux condition, so that $v(z)=\sqrt{v_{0}^{2}-2 g z}$ from equation (2), independent of $a(z)$. 
For instance, for a Case 1 jet shape of constant radius $a_{0}=.003 \mathrm{~m}$, the optimal Case 1 outer tube $R(z)$ occurs when $m=-.149$ (and $R=2.17 \mathrm{~cm}$ ) rather than $m=0$.

Using the jet function $a(z)$ derived from Bernoulli's equation as in (3), rather than the best-fit polynomial matching the Case 2 tube shape, would have made it very difficult to conjecture the optimality condition because the direct relationship between the inner-jet shape and the tube shape would not have been so clear.

\section{The tube optimality condition}

Motivated by this empirical evidence, we consider the proposed optimality condition in detail. We begin by proving its validity, and then obtain a general formula for the multiplying constant and hence for $R(z)$, in terms of the jet shape $a(z)$ and the other parameters including $\delta p$.

We prove the optimality condition by first "discretizing" the problem.

Lemma 1 Let $f(x)$ and $g(x)$ be differentiable functions on some open interval I, with $f^{\prime}(x) / g^{\prime}(x)$ one-to-one on I. Let

$$
Q\left(x_{1}, x_{2}, \ldots, x_{n}\right)=\frac{\sum_{i=1}^{n} b_{i} f\left(x_{i}\right)-\delta}{\sum_{i=1}^{n} b_{i} g\left(x_{i}\right)}
$$


Then the critical points of $Q$ with coordinates in I are exactly points of the form $\left(x_{0}, x_{0}, \ldots, x_{0}\right)$, where $x_{0}$ is a critical point of $\bar{Q}(x)=Q(x, x, \ldots, x)$.

Suppose further that $f$ and $g$ are twice differentiable on $I$, that $b_{i}>0$ for all $i$ and that $\left(f^{\prime \prime}\left(x_{0}\right) g^{\prime}\left(x_{0}\right)-g^{\prime \prime}\left(x_{0}\right) f^{\prime}\left(x_{0}\right)\right) / g\left(x_{0}\right) g^{\prime}\left(x_{0}\right)<0$ at the critical point $\left(x_{0}, x_{0}, \ldots, x_{0}\right)$. Then the critical point is a maximum of $Q$.

Proof: Denote by $F_{i}$ the partial derivative of $F$ with respect to $x_{i}$ throughout.

Let $S=\sum_{i=1}^{n} b_{i} f\left(x_{i}\right)$ and $T=\sum_{i=1}^{n} b_{i} g\left(x_{i}\right)$. Then $S_{j}=b_{j} f^{\prime}\left(x_{j}\right), T_{j}=$ $b_{j} g^{\prime}\left(x_{j}\right)$, and $Q=(S-\delta) / T$. Hence

$$
Q_{j}=\frac{T S_{j}-T_{j}(S-\delta)}{T^{2}}=\frac{T b_{j} f^{\prime}\left(x_{j}\right)-b_{j} g^{\prime}\left(x_{j}\right)(S-\delta)}{T^{2}}
$$

and so

$$
Q_{j}=b_{j} \frac{f^{\prime}\left(x_{j}\right)-Q g^{\prime}\left(x_{j}\right)}{T} .
$$

This is zero when

$$
f^{\prime}\left(x_{j}\right) / g^{\prime}\left(x_{j}\right)=Q\left(x_{1}, x_{2}, \ldots, x_{n}\right),
$$

so $f^{\prime}\left(x_{i}\right) / g^{\prime}\left(x_{i}\right)=f^{\prime}\left(x_{j}\right) / g^{\prime}\left(x_{j}\right)$ for all $i, j$ at any critical point having coordinates in $I$. Because $f^{\prime} / g^{\prime}$ is one-to-one, all $x_{j}$ are equal at such a critical point. 
Note that $\bar{Q}(x)=(f(x)-\delta / s) / g(x)$, where $s=\sum_{i=1}^{n} b_{i}$. Hence the point $\left(x_{0}, x_{0}, \ldots, x_{0}\right)$ is critical if and only if

$$
f^{\prime}\left(x_{0}\right) / g^{\prime}\left(x_{0}\right)=Q\left(x_{0}, x_{0}, \ldots, x_{0}\right)=\bar{Q}\left(x_{0}\right)=\frac{f\left(x_{0}\right)-\delta / s}{g\left(x_{0}\right)},
$$

that is, $f^{\prime}\left(x_{0}\right) g\left(x_{0}\right)-g^{\prime}\left(x_{0}\right) f\left(x_{0}\right)+\delta g^{\prime}\left(x_{0}\right) / s=0$. This happens exactly when $\bar{Q}^{\prime}\left(x_{0}\right)=0$ since $\bar{Q}^{\prime}=\left(f^{\prime} g-g^{\prime} f+g^{\prime} \delta / s\right) / g^{2}$.

Differentiating (14) with respect to $x_{j}$ once more,

$$
\begin{aligned}
Q_{j j} & =\frac{T\left[b_{j} f^{\prime \prime}\left(x_{j}\right)-b_{j} g^{\prime \prime}\left(x_{j}\right) Q-b_{j} g^{\prime}\left(x_{j}\right) Q_{j}\right]-T_{j}\left[b_{j} f^{\prime}\left(x_{j}\right)-b_{j} g^{\prime}\left(x_{j}\right) Q\right]}{T^{2}} \\
& =\frac{b_{j}\left[f^{\prime \prime}\left(x_{j}\right)-g^{\prime \prime}\left(x_{j}\right) Q-g^{\prime}\left(x_{j}\right) Q_{j}\right]}{T}-\frac{b_{j} Q_{j} g^{\prime}\left(x_{j}\right)}{T}
\end{aligned}
$$

which at the critical point $\left(x_{0}, x_{0}, \ldots, x_{0}\right)$ is (using (15))

$$
\begin{aligned}
Q_{j j}\left(x_{0}, x_{0}, \ldots, x_{0}\right) & =\frac{b_{j}\left[f^{\prime \prime}\left(x_{0}\right)-g^{\prime \prime}\left(x_{0}\right) \bar{Q}\left(x_{0}\right)\right]}{s g\left(x_{0}\right)} \\
& =\frac{b_{j}\left[f^{\prime \prime}\left(x_{0}\right)-g^{\prime \prime}\left(x_{0}\right) f^{\prime}\left(x_{0}\right) / g^{\prime}\left(x_{0}\right)\right]}{s g\left(x_{0}\right)} \\
& =\frac{b_{j}\left[f^{\prime \prime}\left(x_{0}\right) g^{\prime}\left(x_{0}\right)-g^{\prime \prime}\left(x_{0}\right) f^{\prime}\left(x_{0}\right)\right]}{s g\left(x_{0}\right) g^{\prime}\left(x_{0}\right)} .
\end{aligned}
$$

Now $b_{j}, s>0$ so this is negative exactly when

$$
\frac{f^{\prime \prime}\left(x_{0}\right) g^{\prime}\left(x_{0}\right)-g^{\prime \prime}\left(x_{0}\right) f^{\prime}\left(x_{0}\right)}{g\left(x_{0}\right) g^{\prime}\left(x_{0}\right)}<0 .
$$


On the other hand, if $i \neq j$, then differentiating (14) with respect to $x_{i}$ gives

$$
Q_{j i}=\frac{-T b_{j} g^{\prime}\left(x_{j}\right) Q_{i}-T_{i}\left[b_{j} f^{\prime}\left(x_{j}\right)-b_{j} g^{\prime}\left(x_{j}\right) Q\right]}{T^{2}},
$$

which is zero at the critical point $\left(x_{0}, x_{0}, \ldots, x_{0}\right)$ from (14). This means that the quadratic form

$$
F\left(x_{1}, x_{2}, \ldots, x_{n}\right)=\sum_{i, j=1}^{n} Q_{i j} x_{i} x_{j}=\sum_{i=1}^{n} Q_{i i} x_{i}^{2},
$$

and so the Hessian matrix of $Q$ is negative definite at $\left(x_{0}, x_{0}, \ldots, x_{0}\right)$, and hence the critical point is a maximum, if (16) holds.

Theorem 2 Suppose we are given the (continuous) jet shape a(z) and all other parameters including $\delta p$. Assume the jet velocity varies according to the conservation of flux condition (1). Then the optimal choice of $R(z)$ is unique and is $R(z)=k a(z)$, where

$$
k=\exp \left[\frac{1}{2}+\frac{1}{2} \text { LambertW }\left(\frac{4 \alpha \mu v_{0} a_{0}^{2}-\delta p}{e \delta p}\right)\right],
$$

and $\alpha=\int_{0}^{L} a(z)^{-4} d z$.

Proof: Let $k(z)=R(z) / a(z)$, defined on the interval $[0, L]$ since $a(z)>0$. Now $R(z)>a(z)$, so $k(z)>1$ on $[0, L]$. The choice of $R(z)$ that optimizes $Q$ 
corresponds to a choice of $k(z)$ defined on $[0, L]$, and having image contained in $I=(1, \infty)$.

Now from equations (1) and (13), we have that $Q=(F-\delta p) /(-G)$, where $F=\int_{0}^{L} b(z) c(k(z)) d z$ and $-G=\int_{0}^{L} b(z) d(k(z)) d z$, with $b(z)=a(z)^{-4}$,

$$
\begin{aligned}
c(k) & =\frac{4 \mu v_{0} a_{0}^{2}\left[2 \log (k)-\left(k^{2}-1\right)\right]}{\left(k^{2}-1\right)^{2}-\left(k^{4}-1\right) \log k} \\
\text { and } \quad d(k) & =\frac{8 \mu}{\pi}\left[\left(k^{4}-1\right)-\frac{\left(k^{2}-1\right)^{2}}{\log k}\right] .
\end{aligned}
$$

We shall think of all parameters as fixed and view $Q$ as a function of $k(z)$. If we restrict to constant $k(z)$, we call the resulting function of a real variable $\bar{Q}(k)$. Thus

$$
\bar{Q}(k)=\frac{c(k)-\delta p / \alpha}{d(k)},
$$

where $\alpha=\int_{0}^{L} a(z)^{-4} d z$.

Now let $P$ be a partition $0=z_{0}<z_{1}<z_{2}<\cdots<z_{n}=L$ of $[0, L]$ and let

$$
Q_{P}\left(x_{1}, x_{2}, \ldots, x_{n}\right)=\frac{\sum_{i=1}^{n} b_{i} c\left(x_{i}\right)-\delta p}{\sum_{i=1}^{n} b_{i} d\left(x_{i}\right)}
$$

where $x_{i}>1$, and $b_{i}=b\left(z_{i}\right)\left(z_{i}-z_{i-1}\right)$ so that the two sums are in fact Riemann sums converging to the corresponding integrals $F$ and $-G$ in $Q$ as the fineness of $P$ tends to zero. Let $f(P)$ denote the fineness of the 
partition $P$, so that $f(P)=\max \left\{\left|z_{i}-z_{i-1}\right|: i=1,2, \ldots, n\right\}$. Thus for any choice of $k(z)$,

$$
Q_{P}\left(k\left(z_{1}\right), k\left(z_{2}\right), \ldots, k\left(z_{n}\right)\right) \rightarrow Q(k(z)) \quad \text { as } \quad f(P) \rightarrow 0 .
$$

Analogous to $\bar{Q}$, we define $\bar{Q}_{P}(k)=Q_{P}(k, k, \ldots, k)$ for $k>1$. Note that

$$
\bar{Q}_{P}(k)=\frac{\sum_{i=1}^{n} b_{i} c(k)-\delta p}{\sum_{i=1}^{n} b_{i} d(k)},
$$

so letting $s_{P}=\sum_{i=1}^{n} b_{i}$,

$$
\bar{Q}_{P}(k)=\frac{c(k)-\delta p / s_{P}}{d(k)} .
$$

Then from (20), for any constant $k$,

$$
\bar{Q}_{P}(k) \rightarrow \bar{Q}(k) \text { as } f(P) \rightarrow 0 .
$$

Note that (21) is (19) with $\alpha$ replaced by $s_{P}$. Then using Maple to differentiate $F(k)=[c(k)-\delta p / \beta] / d(k)$ with respect to $k$ and solving the result set equal to zero gives

$$
k=\exp \left[\frac{1}{2}+\frac{1}{2} \operatorname{LambertW}\left(\frac{4 \beta \mu v_{0} a_{0}^{2}-\delta p}{e \delta p}\right)\right],
$$

the unique critical point of $F$. This is real providing

$$
\frac{4 \beta \mu v_{0} a_{0}^{2}-\delta p}{e \delta p}>-\frac{1}{e}
$$




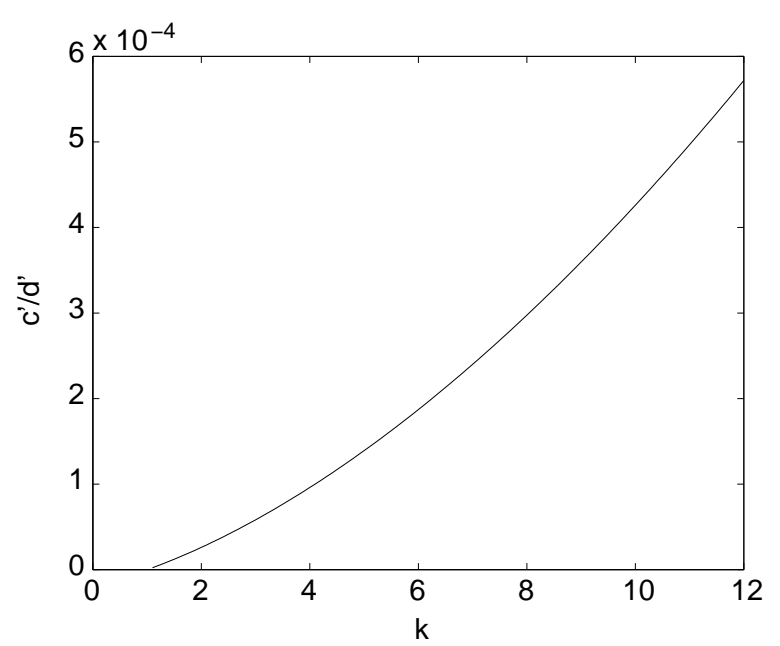

Figure 9: Plot of $h=c^{\prime} / d^{\prime}$

that is, $4 \beta \mu v_{0} a_{0}^{2}>0$, and $\beta=\alpha$ or $s_{P}$ is positive so this is satisfied.

Now $c^{\prime}(x) / d^{\prime}(x)$ is strictly increasing on $I=(1, \infty)$ as seen in the Matlab plot Figure 9 (and this is easily provable in any case) and so is one-to-one. From the lemma, a critical point of $Q_{P}$ with all coordinates in $I$ must have the form $\left(x_{0}, x_{0}, \ldots, x_{0}\right)$ and corresponds exactly to a critical point $x_{0}$ of $\bar{Q}_{P}$. Hence the unique critical point has $x_{0}=k$ as given by (23).

Now $c$ and $d$ are twice differentiable on $I$ and $b_{i}>0$ for all $i$. Letting $h=\left(c^{\prime \prime} d^{\prime}-d^{\prime \prime} c^{\prime}\right)\left(d / d^{\prime}\right)$, we see that $h=\left(c^{\prime} / d^{\prime}\right)^{\prime}\left(d^{\prime} / d\right)$. From Figure 9, 


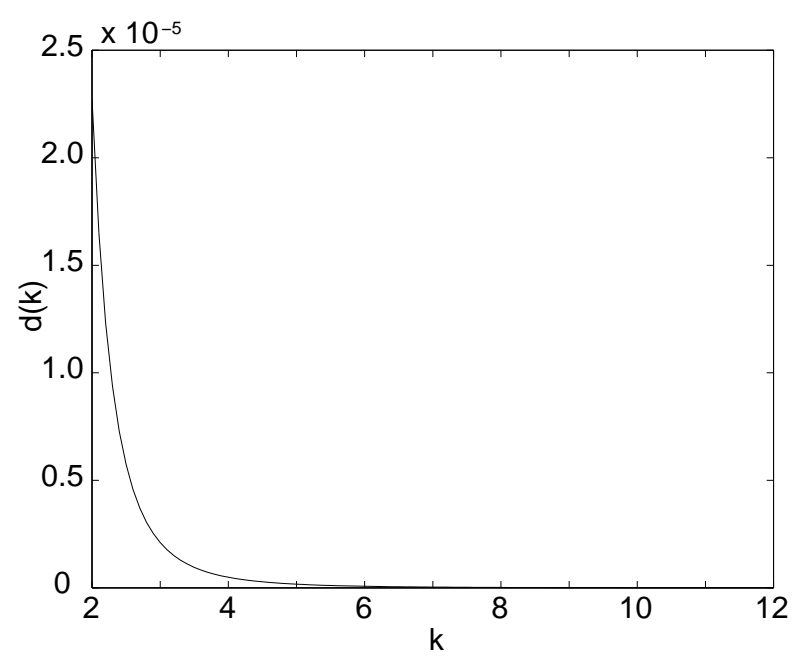

Figure 10: Plot of $d(k)$ versus $k$

$\left(c^{\prime} / d^{\prime}\right)^{\prime}>0$ on $I$, but also $d>0$ and strictly decreasing on $I$, as can be seen from the Matlab plot Figure 10, so $d^{\prime} / d<0$; hence $h<0$ on $I$. Thus the critical point is a maximum of $Q_{P}$ from the lemma; call it $\left(K_{P}, K_{P}, \ldots, K_{P}\right)$. Hence $K_{P}$ is also a maximum of $\bar{Q}_{P}$.

Similarly there is a unique critical point $K$ for $\bar{Q}$, and it will be a maximum because $K_{P}$ is a maximum of $\bar{Q}_{P}$ and because of the similarity between (19) and (21). It remains to show that $k(z)=K$ is optimal for $Q$. 
Because $k$ in (23) is evidently a continuous function of $\beta$, we have

$$
K_{P} \rightarrow K \text { as } f(P) \rightarrow 0
$$

Suppose there exists a choice of $k(z)$ on $[0, L]$ which is non-constant, and gives a value of $Q$ greater than $K$ : thus

$$
Q(k(z))-\bar{Q}(K)=\beta>0 .
$$

Now choose a partition $P$ of $[0, L]$ which is such that $k$ is not constant on the $z_{i}$, and is sufficiently fine that

$$
\begin{gathered}
\left|Q_{P}\left(k_{1}, k_{2}, \ldots, k_{n}\right)-Q(k(z))\right|<\frac{\beta}{4}, \\
\left|\bar{Q}_{P}\left(K_{P}\right)-\bar{Q}_{P}(K)\right|<\frac{\beta}{4}
\end{gathered}
$$

and

$$
\left|\bar{Q}_{P}(K)-\bar{Q}(K)\right|<\frac{\beta}{4} .
$$

Taken separately, the first of these is possible by (20), the second by (24) and the continuity of $\bar{Q}_{P}$ on $I$ with respect to $k$, and the third by (22); to ensure all three hold at once for $P$, it is necessary that $f(P)$ be no bigger than the minimum of the three $f(P)$ 's needed to guarantee each separately. Then

$$
\begin{aligned}
& Q_{P}\left(k_{1}, k_{2}, \ldots, k_{n}\right)-Q_{P}\left(K_{P}, K_{P}, \ldots, K_{P}\right) \\
= & Q_{P}\left(k_{1}, k_{2}, \ldots, k_{n}\right)-\bar{Q}_{P}\left(K_{P}\right)
\end{aligned}
$$




$$
\begin{aligned}
& >\left[Q(k(z))-\frac{\beta}{4}\right]-\left[\bar{Q}_{P}(K)+\frac{\beta}{4}\right] \quad \text { by }(26) \text { and }(27) \\
& =Q(k(z))-\frac{\beta}{2}-\bar{Q}_{P}(K) \\
& >Q(k(z))-\frac{\beta}{2}-\left[\bar{Q}(K)+\frac{\beta}{4}\right] \quad \text { by }(28) \\
& =Q(k(z))-\bar{Q}(K)-\frac{3 \beta}{4} \text { by }(25) \\
& =\frac{\beta}{4},
\end{aligned}
$$

contradicting the optimality of $\left(K_{P}, K_{P}, \ldots, K_{P}\right)$ for $Q_{p}$. Hence there is no non-constant $k(z)$ giving a higher value of $Q$ than $K$. Certainly there is no constant value since $K$ was the largest constant value, so $K$ is in fact the optimal choice of $k(z)$ to optimize $Q$, and so there is indeed exactly one optimal tube, $R(z)=k a(z)$, with $k$ as in the theorem statement.

\section{Optimizing the outer tube for various pres- sure differences}

The job of finding the optimal tube given $a(z)$ was completed by the previous section: if $R(z)=k a(z)$ ( $k$ some constant), then (23) gives $k$ as a function of $\alpha, \delta p$ and the other parameters. Note that the value of $k$ is not directly 


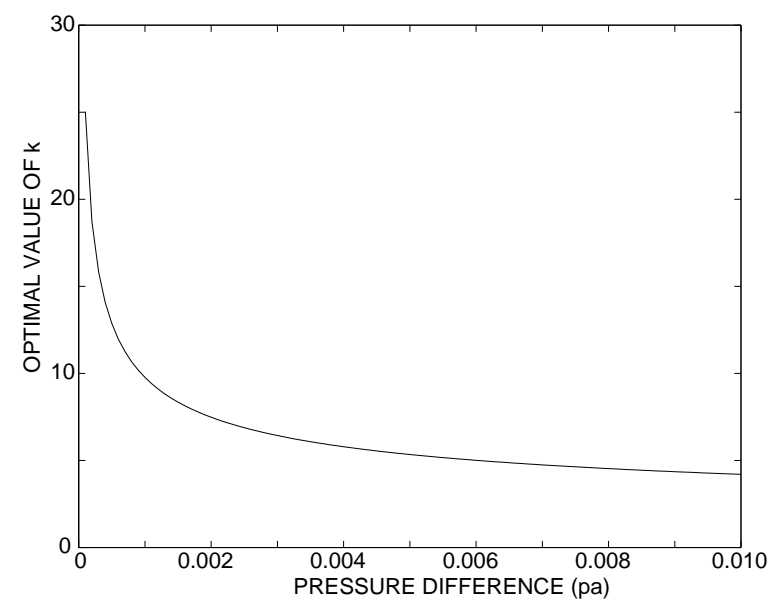

Figure 11: Optimal multiplying constant $k$ versus $\delta p$ for actual jet

dependent on $a(z)$ but rather on $\alpha$, the integral of $a(z)^{-4}$ over $[0, L]$. (Of course, the optimal $R(z)$ is dependent on $a(z)$.) Also note that the expression for $k$ in (23) has a very similar form to (11), obtained in the seemingly very special case in which the inner jet was assumed to be a cylinder. In fact (11) is obtained from (23) by replacing $\alpha$ by $L / a_{0}^{4}$ and $p$ by $L d p / d z$.

With the usual values for the parameters and the actual jet shape

$$
a(z)=\left(\frac{v_{0} a_{0}^{2}}{\left(v_{0}^{2}-2 g z\right)^{1 / 2}}\right)^{1 / 2}
$$

as in (3), it turns out that $\alpha=4.38 \times 10^{8} \mathrm{~m}^{-3}$ from Matlab. (This value 


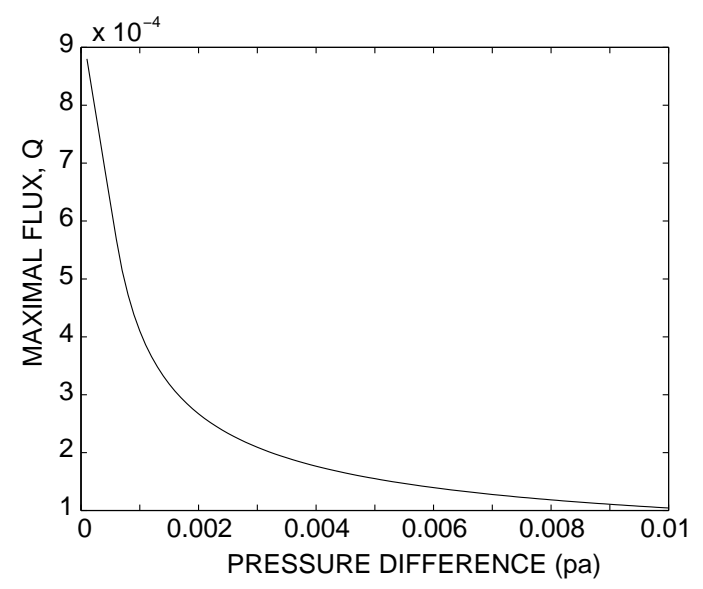

Figure 12: Flux versus pressure difference $\delta p$ for actual jet

was used for all Matlab plots in the theorem proof.) A plot of $k$ against $\delta p$ for this case (or indeed any other inner jet $a(z)$ for which $\alpha$ has this value) appears in Figure 11. Note the similarity to Figure 5 in Section 4 which it generalises.

As in Section $4, k$ increases without limit as $\delta p \rightarrow 0$, but drops away to some constant value as $\delta p \rightarrow \infty$. From (23), the value approached is

$$
\exp \left[\frac{1}{2}+\frac{1}{2} \operatorname{LambertW}\left(-\frac{1}{e}\right)\right]=1,
$$

consistent with the findings of the fourth section. 


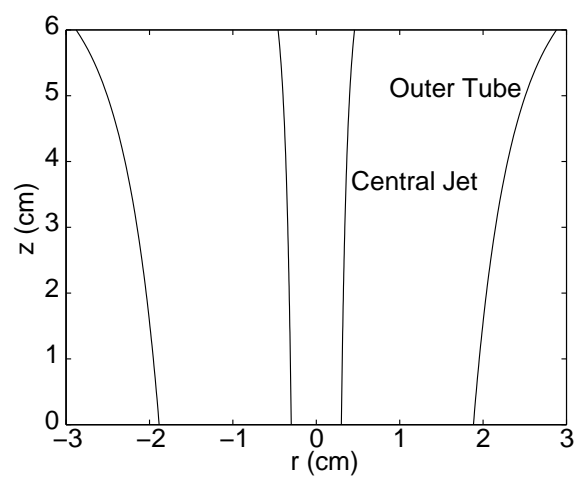

Figure 13: Optimal tube size and shape for actual jet

A plot of optimal $Q$ against $\delta p$ is given in Figure 12 .

For the case in which $\delta p=3.2 \times 10^{-3} \mathrm{~Pa}$, the optimum flow occurs when $k=6.28$, with $Q=2.01 \times 10^{-4} \mathrm{~m}^{3} / \mathrm{s}$, consistent with earlier findings. This optimal arrangement is depicted in Figure 13.

It is important to note that for a tube with given average radius, the optimal shape is not necessarily just a multiple of the jet shape. It is only at the global maximum of flux that this is true. Figure 8 shows the effects of both $m$ and $R_{0}$ on the flux, and it is clear that the maximum is not correlated directly to either of these two parameters, but rather to some combination. 


\section{Conclusions}

We have shown that the optimal shape of the inner tube is defined by a function that is a constant multiple of the function defining the jet shape. Moreover, this constant can be explicitly given as a function of the pressure difference across the tube $\delta p$, two constants associated with the jet shape $a(z)$ (namely $a_{0}=a(0)$ and $\alpha=\int_{0}^{L} a^{-4} d z$ ), and the initial jet velocity, $v_{0}$. However, it is not true that the optimal shape for a tube of some given average radius is simply the shape of the central jet, it is only true at a particular radius. Fortunately, Figure 8 shows that the flux is not particularly sensitive to small variations in the parameters of the tube close to the optimal value, and so we would expect that a shape close to optimal would still be reasonably efficient in the delivery of the aerosol.

Note that no consideration has been made of the effect that the tube shape or radius has on the pressure in the upper and lower chambers. However, it would seem likely that the pressure differential would increase as the flux increases, slightly off-setting the benefits of the optimal tube. This is a more difficult problem due to the presence of the aerosol mist and may change the results slightly, although it is unlikely that the general finding will change. 


\section{References}

[1] Batchelor, G.K., An Introduction to Fluid Mechanics, Cambridge University Press, 1967. E43

[2] Hocking, G., \& Fulford, G., 'The Sheiman Ultrasonic Nebulizer', Proceedings of 1998 Mathematics-in-Industry Study Group, Ed. J. Hewitt. E39, E43

[3] Maple V Release 5, copyright 1981-1997 by Waterloo Maple Inc. E50 